\title{
Silencing of hypoxia-inducible adrenomedullin using RNA interference attenuates hepatocellular carcinoma cell growth in vivo
}

\author{
FENBAO LI, RUIMIN YANG, XIZHONG ZHANG, AIGUANG LIU, YONGLI ZHAO and YINGCHANG GUO \\ Department of Interventional Therapy, First Affiliated Hospital of Xinxiang Medical University, \\ Xinxiang, Henan 453100, P.R. China
}

Received August 6, 2013; Accepted April 14, 2014

DOI: $10.3892 / \mathrm{mmr} .2014 .2320$

\begin{abstract}
Adrenomedullin (ADM) is an angiogenic peptide that has been shown to increase the risk of endometrial hyperplasia and to promote tumor cell survival following hypoxia. ADM may induce microvessel proliferation and partially decrease hypoxia in solid tumors, thus contributing to the proliferation of tumor cells, as well as tumor invasion and metastasis. However, the impact of hypoxia-induced ADM expression on hepatocellular carcinoma (HCC) cells requires further elucidation. In the present study it was found that the levels of ADM mRNA in tumor tissue from patients with HCC were significantly increased compared with the mRNA levels in adjacent non-tumorous liver tissue. Under hypoxic conditions, the mRNA and protein levels of ADM, as well as those of the cancer-promoting genes vascular endothelial growth factor and hypoxia-inducible factor $1 \alpha$, were significantly elevated in a time-dependent manner in three human HCC cell lines. In addition, knockdown of ADM expression using short hairpin RNA (shRNA) in SMMC-7721 cells resulted in apoptosis that was not observed in untransfected cells. Furthermore, combined treatment with cisplatin and ADM-shRNA significantly decreased tumor growth in vivo compared with treatment with cisplatin or ADM-shRNA alone. These data demonstrate that ADM acts as a critical promoter of cell cycle progression in HCC and that the inhibition of ADM may be an effective interventional therapeutic strategy in HCC.
\end{abstract}

\section{Introduction}

Hepatocellular carcinoma (HCC) is a complex disease initiated by chronic hepatitis $\mathrm{B}$ and $\mathrm{C}$ infections, exposure

Correspondence to: Dr Ruimin Yang, Department of Interventional Therapy, First Affiliated Hospital of Xinxiang Medical University, 88 Jiankang Road, Xinxiang, Henan 453100, P.R. China

E-mail: ruim_yang@163.com

Key words: adrenomedullin, interventional therapy, hepatocellular carcinoma, shRNA, tumor growth to environmental chemicals or alcohol or metabolic liver diseases. These interactions lead to a multistep carcinogenic process, although the molecular and cellular mechanisms that underlie HCC pathogenesis remain to be elucidated (1). It is widely accepted that tumor cell growth is an important factor in controlling tumor cell cycle progression, resulting from genetic anomalies in cancer cells that affect numerous cell growth regulatory pathways as well as tumor cell interactions with microenvironmental factors. Hypoxia has been proposed to participate in the genesis and progression of HCC as this tumor type is typically accompanied by neovascularization and hyper-vascularity (2). There is evidence that hypoxia stimulates HCC cell growth and the expression of specific target genes such as hexokinase II (3).

Angiogenesis and the production of angiogenic factors are essential for tumor growth, invasion and metastasis (4). Tumor angiogenesis has been associated with an imbalance in the equilibrium between positive and negative regulators (5) and is primarily triggered by the release of endothelial cell-specific growth factors by neoplastic cells that stimulate the growth of host blood vessels (6). This imbalance depends on an increased production of one or more positive regulators of angiogenesis, including vascular endothelial growth factor (VEGF), which are hypoxia-inducible factor 1 (HIF-1)-dependent genes that are exported from tumor cells, mobilized from the extracellular matrix or released from host cells recruited to the tumor site (7). Tumor angiogenesis is complex and comprises a number or processes alongside the upregulation of angiogenic activity, and has thus been regarded as the result of a net balance of positive and negative regulators (5).

Adrenomedullin (AMD) is an angiogenic peptide that was originally isolated from extracts of human pheochromocytomas (8). This peptide has also been shown to be a mitogen and a hypoxia survival factor of tumor cells that promotes tumor proliferation and inhibits apoptosis through a number of signaling pathways (9). The detection of high levels of AMD expression in various types of cancer, including prostatic carcinoma (10), cervical cancer (11) and HCC (12), suggests that this peptide is involved in tumor growth.

To further determine the role of ADM in HCC, the expression of this protein in HCC cells and normal tissue was 
compared in the present study. Intracellular levels of ADM were assessed in tissue samples from high-grade tumors and compared with those from low-grade tumors or normal tissue. ADM has been reported to be involved in vascular invasion and $\mathrm{N}$-cadherin expression due to hypoxia through a pathway of Akt activation (12). In the present study, the secretion of the ADM peptide by HCC cells under normal and hypoxic conditions was investigated. Furthermore, it was investigated whether decreasing ADM expression using RNA interference would impact the growth of HCC cells, and the interventional therapeutic efficacy of a combination of cisplatin and ADM-knockdown on tumor growth was assessed in vivo.

\section{Materials and methods}

Patient population. A total of 64 patients with HCC who underwent curative liver resection at the First Affiliated Hospital of Xinxiang Medical University (Xinxiang, China), between April 2010 and July 2012 were enrolled in the present study. The participants provided their written informed consent prior to participation, and the study protocol was approved by the Institutional Review Board of the Cancer Institute of Xinxiang Medical University. The present clinical investigation was conducted according to the principles expressed in the Declaration of Helsinki. The mean age of the patients was 50 years (range, 38-69 years), and 48 of them were male. Samples of normal liver tissue near the tumors were collected for use as controls. All the samples were classified by two experienced pathologists based on histology and cytology using the Edmondson-Steiner grading system (13). The patients with HCC were divided into four groups as follows: 18 cases were stage I, 24 cases were stage II, 13 cases were stage III and nine were stage IV. None of the patients received any preoperative anticancer treatment. The HCC and non-tumorous liver tissue samples were excised and snap-frozen in liquid nitrogen for subsequent RNA and protein extraction.

Cell culture and reagents. Human HCC HepG2, SMMC-7721 and Bel-7402 cells were obtained from the American Type Culture Collection (Rockville, MD, USA). These cell lines were cultured in RPMI-1640 supplemented with $15 \%$ fetal bovine serum under standard culture conditions $\left(20 \% \mathrm{O}_{2}\right.$ and $5 \% \mathrm{CO}_{2}$ at $37^{\circ} \mathrm{C}$ ). A primary anti-ADM antibody was obtained from Abcam (Cambridge, MA, USA). The anti-VEGF and anti-HIF- $1 \alpha$ antibodies were purchased from Santa Cruz Biotechnology, Inc. (Santa Cruz, CA, USA). An anti-GAPDH antibody was purchased from Cell Signaling Technology, Inc. (Beverly, MA, USA). All of the chemicals were purchased from Sigma-Aldrich (St. Louis, MO, USA).

Induction of hypoxia in HCC cells. HepG2, Bel-7402 and SMMC-7721 cells were seeded in six-well plates at a density of $2 \times 10^{4}$ cells/well, allowing for an exponential growth rate. Each cell line was divided into four groups: $6 \mathrm{~h}$ hypoxia, $12 \mathrm{~h}$ hypoxia, $24 \mathrm{~h}$ hypoxia and a normal control group, and each group included three triplicate wells. Initially, the 24-h hypoxia groups were exposed to hypoxic conditions $\left(1 \% \mathrm{O}_{2}\right.$, $5 \% \mathrm{CO}_{2}$ and $94 \% \mathrm{~N}_{2}$ at $37^{\circ} \mathrm{C}$ ). After 12 and $18 \mathrm{~h}$ of incubation, respectively, the 12- and 6-h hypoxia groups were exposed to the same hypoxic conditions. The normal control group was incubated under normal culture conditions in parallel. After $24 \mathrm{~h}$, the cells were digested with trypsin, and total RNA and protein were extracted for further assays.

Generation of an SMMC-7721 cell line stably expressing ADM-short hairpin RNA (shRNA). SMMC-7721 cells from the sixth passage were seeded in six-well plates ( $1 \times 10^{5}$ cells/well) and allowed to adhere for $24 \mathrm{~h}$ prior to transfection. The cells were transfected with plasmids containing shRNA directed against human ADM (ADM-shRNA; Santa Cruz Biotechnology, Inc.) or a non-targeting vector-control shRNA (NC-shRNA; Santa Cruz Biotechnology, Inc.). In addition, the green fluorescence protein control plasmid (Santa Cruz Biotechnology, Inc.) was used to monitor and optimize transfection efficiency. At $72 \mathrm{~h}$ post-transfection, puromycin $(7.0 \mathrm{mg} / \mathrm{ml})$ was added to the culture medium for selection and further characterization. The transfection efficiency of SMMC-7721 cells expressing ADM-shRNA was assessed using flow cytometry.

Western blot analysis. Cells or tissue samples were lysed for $20 \mathrm{~min}$ on ice and centrifuged at $14,000 \mathrm{x}$ g for $10 \mathrm{~min}$ at $4^{\circ} \mathrm{C}$. The supernatant was collected, and the protein concentration was determined using a Bradford assay (Bio-Rad, Hercules, CA, USA). Proteins were separated using 12\% SDS-PAGE and transferred to a polyvinylidene fluoride membrane (Bio-Rad). The membranes were then blocked with 5\% skimmed milk for $1 \mathrm{~h}$ and incubated overnight with the following primary antibodies (all diluted 1:1,000): anti-ADM (ab69117; Abcam), anti-VEGF (sc-152; Santa Cruz Biotechnology, Inc.), anti-HIF-1 $\alpha$ (sc-10790; Santa Cruz Biotechnology, Inc.) and anti-GAPDH (2118; Cell Signaling Technology, Inc.). The membranes were then incubated with a secondary antibody for $1.5 \mathrm{~h}$ at room temperature. Membrane-bound antibodies were visualized using a chemiluminescent substrate (Pierce, Rockford, IL, USA) and exposed to X-OMAT film (Kodak, Rochester, NY, USA). Immunoreactive protein bands were quantified by densitometry using QuantityOne software (Bio-Rad).

$R N A$ extraction and quantitative polymerase chain reaction $(q P C R)$. Total RNA was extracted from cells and tissues using the RNAiso Plus Kit (Takara, Dalian, China). A total of $1 \mu \mathrm{g}$ total RNA was used for first-strand DNA synthesis using the PrimeScript RT kit reagents (Takara), and qPCR was performed using primers specific for the respective human genes and the SYBR ${ }^{\circledR}$ Green Premix kit (Takara). For each primer pair, the annealing temperature was optimized by gradient PCR. The expression of each target mRNA relative to the GAPDH mRNA was calculated based on $\mathrm{Ct}$ as $2^{-\Delta(\Delta \mathrm{Ct})}$. The primer sequences used are shown in Table I.

Flow cytometry. Apoptosis in SMMC-7721 cells was assessed using annexin V/propidium iodide (PI) staining. Following $72 \mathrm{~h}$ of incubation, SMMC-7721 cells stably expressing ADM-shRNA were stained with annexin V-fluorescein isothiocyanate and PI using an Apoptosis Detection kit (Sigma-Aldrich) according to the manufacturer's instructions. 
Table I. Primers used for quantitative polymerase chain reaction.

\begin{tabular}{|c|c|c|}
\hline Gene & Forward primer & Reverse primer \\
\hline $\mathrm{ADM}$ & 5'-acttggcagatcactctcttagca-3' & 5'-atcagggcgacggaaacc-3' \\
\hline VEGF & 5'-gcacccatggcagaagg-3' & $5^{\prime}$-ggggtacccetcaccgcetcggettgtc- $3^{\prime}$ \\
\hline $\mathrm{HIF}-1 \alpha$ & 5'-gaaagcgcaagtcctcaaag-3' & 5'-tgggtaggagatggagatgc-3' \\
\hline GAPDH & 5'-caaattccatggcaccgtc-3' & 5'-cccatctgattttggaggga-3' \\
\hline
\end{tabular}

ADM, adrenomedullin; VEGF, vascular endothelial growth factor; HIF-1 $\alpha$, hypoxia-inducible factor $1 \alpha$.

SMMC-7721 cells transfected with non-specific vector-shRNA were used as a vehicle control. Apoptotic cells were quantified using a FACSCalibur ${ }^{\mathrm{TM}}$ flow cytometer (BD Biosciences, Franklin Lakes, NJ, USA).

Animal studies. All animal experiments in the present study were performed in accordance with a protocol approved by the Institutional Animal Care and Use Committee. A total of 48 six-week-old male BALB/c mice were randomly divided into six groups [cisplatin plus ADM-shRNA, cisplatin plus vector, cisplatin plus phosphate-buffered saline (PBS), saline plus ADM-shRNA, saline plus vector and saline plus PBS; $\mathrm{n}=8$ for each group] to receive subcutaneous injections of $0.1 \mathrm{ml}$ SMMC-7721 tumor cells into the right flank. The first three groups received intratumoral administrations of $10 \mathrm{mg} / \mathrm{kg}$ body weight of either ADM-shRNA, NC-shRNA or an equal volume of PBS following intraperitoneal injections of cisplatin ( $5 \mathrm{mg} / \mathrm{kg}$ body weight). The remaining three groups were treated with saline rather than cisplatin as controls. Either shRNA, cisplatin, saline or PBS was injected every three days for 28 days. The mean tumor volumes of the primary tumors were measured in three dimensions $(a, b$ and $c$ ) every three days with a caliper and calculated according to the following formula: $a \times b \times c \times 0.52$. At the end of the experiment, the mice were sacrificed, tumors were harvested and tumor protein levels were assessed using western blot analysis.

Statistical analysis. All of the in vitro experimental data represent at least three independent experiments and are expressed as the mean \pm standard error of the mean. Significant differences were evaluated using a one-way analysis of variance (ANOVA) followed by Bonferroni multiple comparisons tests using the commercially available SPSS software package (SPSS, Inc., Chicago, IL, USA). The degrees of freedoms of the 'Treatment' and 'Residue' for the F-value were also reported. Differences were considered to be statistically significant when the P-value was $<0.05$.

\section{Results}

ADM expression in human HCC tissue samples. To investigate the adaptive upregulation of ADM in HCC cells, endogenous expression levels of ADM were assessed in normal and tumorous (stage I, II, III and IV) tissues. ADM protein levels were markedly elevated in tumorous tissue compared with normal liver tissue $\left(\mathrm{F}_{4,77}=6.85 ; \mathrm{P}<0.01\right)$. ADM levels in stage II,

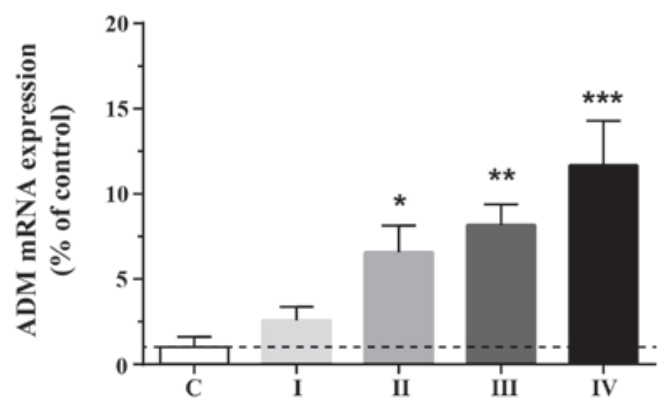

Figure 1. Quantitative polymerase chain reaction detection of ADM mRNA levels in 50 samples of hepatocellular carcinoma tissue and adjacent normal liver tissue. Eighteen cases were classified as stage I, 24 were stage II, 13 were stage III, and nine were stage IV. GAPDH was used as an internal control. ADM mRNA levels in adjacent normal liver tissue were set as $100 \%$ for normalization. Data are presented as the mean \pm standard error of the mean. ${ }^{*} \mathrm{P}<0.05,{ }^{* *} \mathrm{P}<0.01$ and ${ }^{* * *} \mathrm{P}<0.0001$ compared with normal controls. ADM, adrenomedullin; C, control.

III and IV cancer tissue were significantly higher than those in the normal controls (Fig. 1), suggesting that the expression of ADM is upregulated in human HCC tissue and that this may promote tumor growth.

Hypoxia-induced ADM, VEGF and HIF-1 $\alpha$ expression in HCC cells. To investigate whether hypoxia activates ADM expression in HCC cells, the expression of ADM and other cancer promoting genes, such as VEGF and HIF-1 $\alpha$, was assessed following exposure to hypoxic conditions. Western blot analysis showed that the protein expression levels of ADM, VEGF and HIF-1 $\alpha$ increased significantly in the three different HCC cell lines with increasing time under hypoxic conditions (Fig. 2A). The levels of ADM, VEGF and HIF-1 $\alpha$ transcripts correlated with the protein expression levels (Fig. 2B). Among the three types of human HCC cell lines that were tested, SMMC-7721 cells appeared to be the most sensitive to hypoxic stress. The statistical results of these data are summarized in Table II.

Generation of SMMC-7721 cells that stably express $A D M$-shRNA. Accumulating evidence suggests that HCC development is closely associated with constitutive upregulation of ADM $(12,14,15)$. As SMMC-7721 cells are sensitive to hypoxic stress, a lentiviral ADM-shRNA was transfected into SMMC-7721 cells. The transfection efficiency following puromycin selection was determined to be $85.9 \%$ using flow cytometry (Fig. 3A). According to qPCR and western 

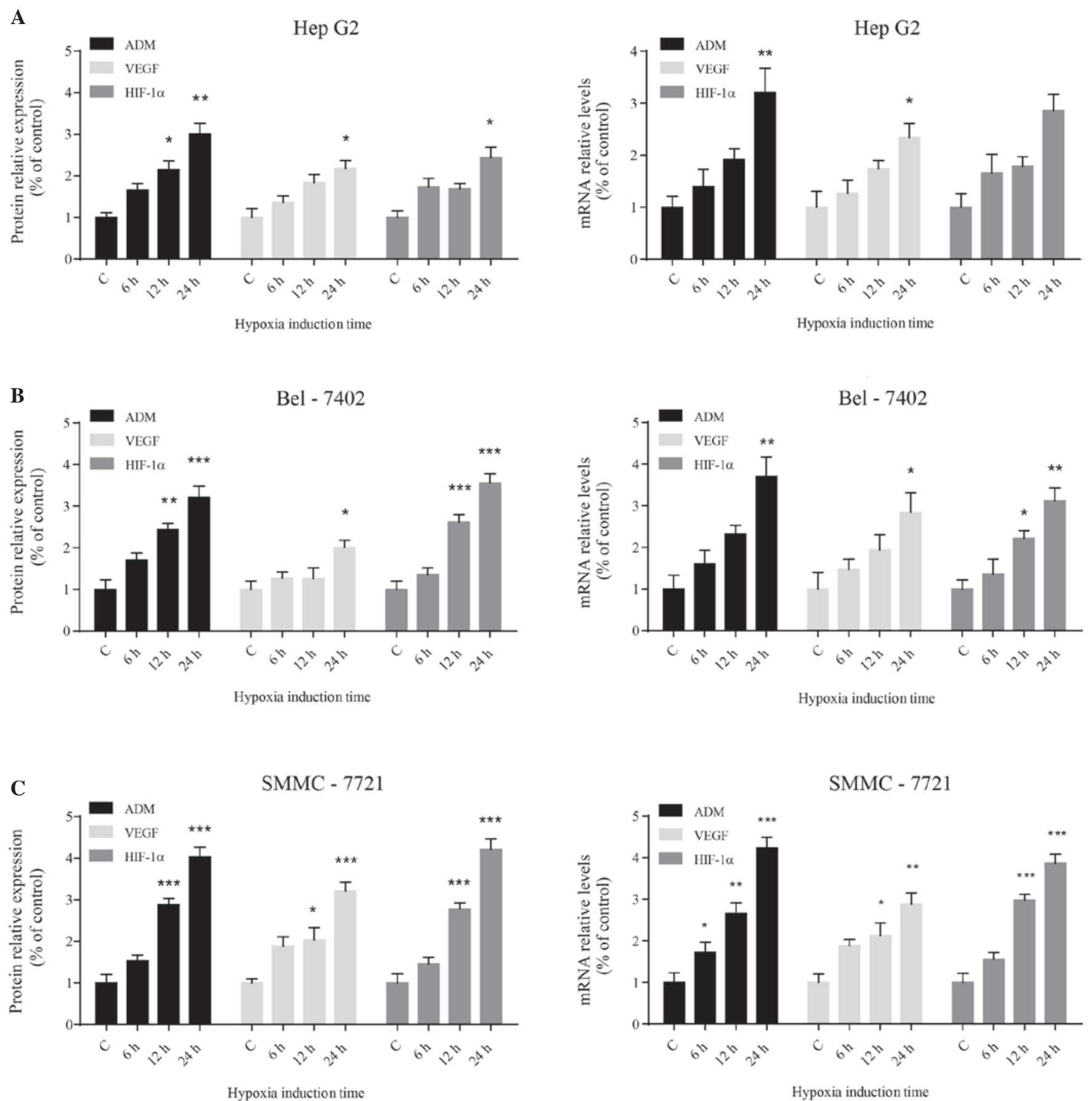

Figure 2. Relative protein and mRNA levels of ADM, VEGF and HIF-1 $\alpha$ following hypoxia treatment in (A) HepG2, (B) Bel-7402 and (C) SMMC-7721 cells . The normal control group was incubated under normal culture conditions in parallel. Data are presented as the mean \pm standard error of the mean. " $\mathrm{P}<0.05$, ${ }^{* *} \mathrm{P}<0.01$ and ${ }^{* * *} \mathrm{P}<0.0001$ compared with normal controls. ADM, adrenomedullin; VEGF, vascular endothelial growth factor; HIF-1 $\alpha$, hypoxia-inducible factor $1 \alpha$; , control.

blot analysis, both ADM mRNA $\left(\mathrm{F}_{2,15}=12.94 ; \mathrm{P}=0.0005\right)$ and protein $\left(\mathrm{F}_{2,15}=18.43 ; \mathrm{P}<0.0001\right)$ levels in $\mathrm{SMMC}-7721$ cells decreased significantly following transfection with ADM-shRNA (Fig. 3B). The relative ADM mRNA expression in cells transfected with ADM-shRNA was $0.23 \pm 0.08$-fold lower than that in normal SMMC-7721 cells $(\mathrm{P}<0.0001)$. The expression of ADM was not observed to be different between vector-control-transfected and untransfected cells. These results revealed that the ADM-shRNA used in the present study successfully knocked down ADM in SMMC-7721 cells.
ADM-knockdown promotes apoptosis in SMMC-7721 cells. After $72 \mathrm{~h}$ of cell growth, significantly increased levels of cell death were observed in SMMC-7721 cells stably expressing ADM-shRNA (mean, $37.34 \pm 0.71 \%$ apoptotic cells) as compared with the untransfected cells (mean, $5.44 \pm 0.31 \%$ apoptotic cells $)\left(\mathrm{F}_{2,14}=12.13 ; \mathrm{P}<0.0035\right.$; post-hoc, $\left.\mathrm{P}=0.0021\right)$ (Fig. 4). The apoptosis observed in the vector-control-transfected group (mean, $9.66 \pm 0.40 \%$ apoptotic cells) was slightly increased compared with that observed in the untransfected SMMC-7721 cells; however, this difference failed to reach statistical significance $(\mathrm{P}=0.0642)$. 
Table II. Hypoxia-induced ADM, VEGF and HIF-1 expression in hepatocellular carcinoma cells.

\begin{tabular}{|c|c|c|c|c|}
\hline \multirow[b]{2}{*}{ Gene } & \multicolumn{2}{|c|}{ Protein } & \multicolumn{2}{|c|}{ mRNA } \\
\hline & F-value $(3,8)$ & P-value & F-value $(3,8)$ & P-value \\
\hline \multicolumn{5}{|l|}{ HepG2 } \\
\hline ADM & 19.56 & $0.0005^{\mathrm{a}}$ & 9.054 & $0.0060^{\mathrm{a}}$ \\
\hline VEGF & 14.09 & $0.0015^{\mathrm{a}}$ & 5.370 & $0.0256^{\mathrm{b}}$ \\
\hline HIF- $1 \alpha$ & 9.280 & $0.0055^{\mathrm{a}}$ & 7.288 & $0.0112^{\mathrm{b}}$ \\
\hline \multicolumn{5}{|l|}{ Bel-7402 } \\
\hline ADM & 21.19 & $0.0004^{\mathrm{a}}$ & 11.51 & $0.0028^{\mathrm{a}}$ \\
\hline VEGF & 4.761 & $0.0345^{\mathrm{b}}$ & 4.304 & $0.0439^{b}$ \\
\hline HIF- $1 \alpha$ & 37.84 & $<0.0001^{\mathrm{c}}$ & 11.70 & $0.0027^{\mathrm{a}}$ \\
\hline \multicolumn{5}{|c|}{ SMMC-7721 } \\
\hline ADM & 55.29 & $<0.0001^{\mathrm{c}}$ & 26.01 & $0.0002^{\mathrm{a}}$ \\
\hline VEGF & 16.76 & $0.0008^{\mathrm{a}}$ & 10.66 & $0.0036^{\mathrm{a}}$ \\
\hline HIF-1 $\alpha$ & 53.46 & $<0.0001^{\mathrm{c}}$ & 49.90 & $<0.0001^{\mathrm{c}}$ \\
\hline
\end{tabular}

${ }^{\mathrm{a}} \mathrm{P}<0.01,{ }^{\mathrm{b}} \mathrm{P}<0.05$ and ${ }^{\mathrm{c}} \mathrm{P}<0.0001$. Significance for the main factor was revealed by one-way analysis of variance analysis. ADM, adrenomedullin; VEGF, vascular endothelial growth factor; HIF-1 $\alpha$, hypoxia-inducible factor $1 \alpha$.

Table III. Rate of tumor inhibition 28 days after SMMC-7721 cell implantation.

\begin{tabular}{lcc}
\hline Group $(\mathrm{n}=8)$ & Tumor volume $\left(\mathrm{mm}^{3}\right)$ & Inhibition rate $(\%)$ \\
\hline Saline & & \\
PBS & $1720 \pm 38.33$ & - \\
Vector & $1599 \pm 36.98$ & 9.20 \\
ADM-shRNA & $884 \pm 11.15$ & 49.45 \\
Cisplatin & & \\
PBS & $1050 \pm 75.30$ & 39.21 \\
Vector & $955 \pm 25.37$ & 45.70 \\
ADM-shRNA & $501 \pm 16.60$ & $71.39^{\mathrm{a}}$ \\
\hline
\end{tabular}

${ }^{\mathrm{a}} \mathrm{P}<0.05$ when compared with mice treated with cisplatin $+\mathrm{PBS}$. PBS, phosphate-buffered saline; ADM, adrenomedullin; shRNA, short hairpin RNA.

Therapeutic efficacy of the combination of cisplatin and ADM-knockdown on tumor growth in vivo. To elucidate whether ADM-shRNA and cisplatin are able to act synergistically in vivo, SMMC-7721 cells were transplanted into mice to generate subcutaneous tumors. In conjunction, a subset of the mice was treated with cisplatin. Tumor growth was significantly inhibited in mice injected with cisplatin (Fig. 5A). When cisplatin treatment was combined with ADM-shRNA delivery, tumor growth inhibition was significantly higher than that following treatment with cisplatin alone (71.39 versus $39.21 \%$; Bonferroni post-hoc, $\mathrm{P}<0.05)$. Tumor inhibition rates are shown in Table III. Injection of the ADM-shRNA plasmid alone also suppressed tumor growth $(49.45 \%)$. The tumor volume did not differ significantly between controls treated with vector-shRNA and control mice injected with PBS. Analysis of the proteins expressed in the tumors showed a marked decrease in ADM (Fig. 5B).
A
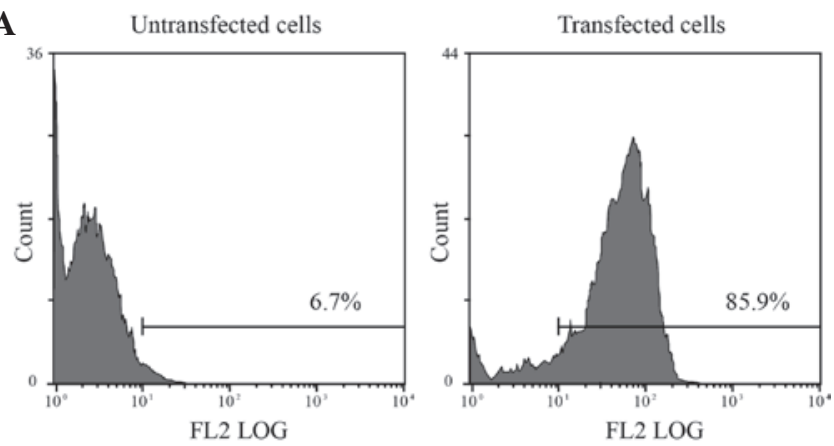

B
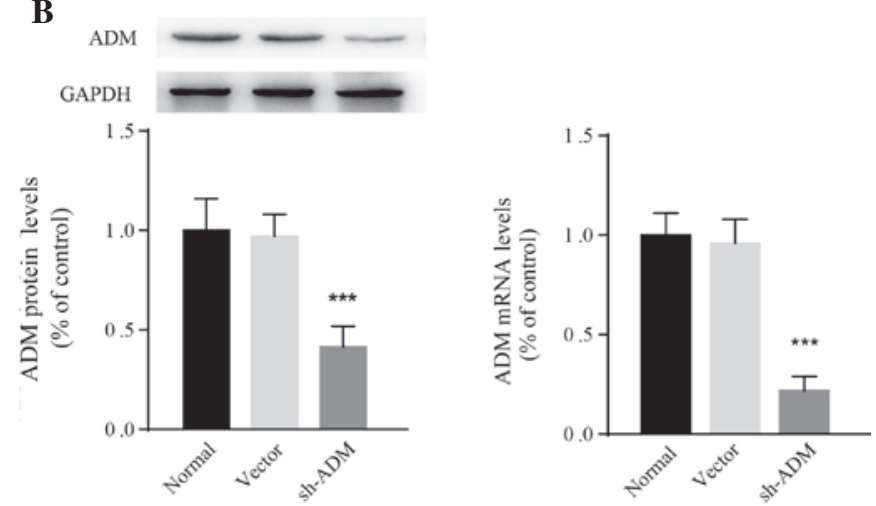

Figure 3. SMMC-7721 cells were transfected with plasmids expressing ADM-shRNA. (A) Following selection with puromycin, the transfection efficacy in the negative control cells was $6.7 \%$ (left) compared with $85.9 \%$ in the cells stably expressing ADM-shRNA (right), as measured using flow cytometry. (B) Gene silencing of ADM by ADM-shRNA caused decreased expression of protein (left) and mRNA (right) levels of ADM. The data are presented as the mean \pm standard error of the mean. ${ }^{*} \mathrm{P}<0.05,{ }^{* *} \mathrm{P}<0.01$ and ${ }^{* * * *} \mathrm{P}<0.0001$ compared with normal controls. ADM, adrenomedullin; shRNA, short hairpin RNA.

Two-way ANOVA revealed that cisplatin $\left(\mathrm{F}_{1,30}=8.37 ; \mathrm{P}<0.01\right)$ and ADM-shRNA $\left(\mathrm{F}_{2,30}=27.59 ; \mathrm{P}<0.0001\right)$ exerted significant 

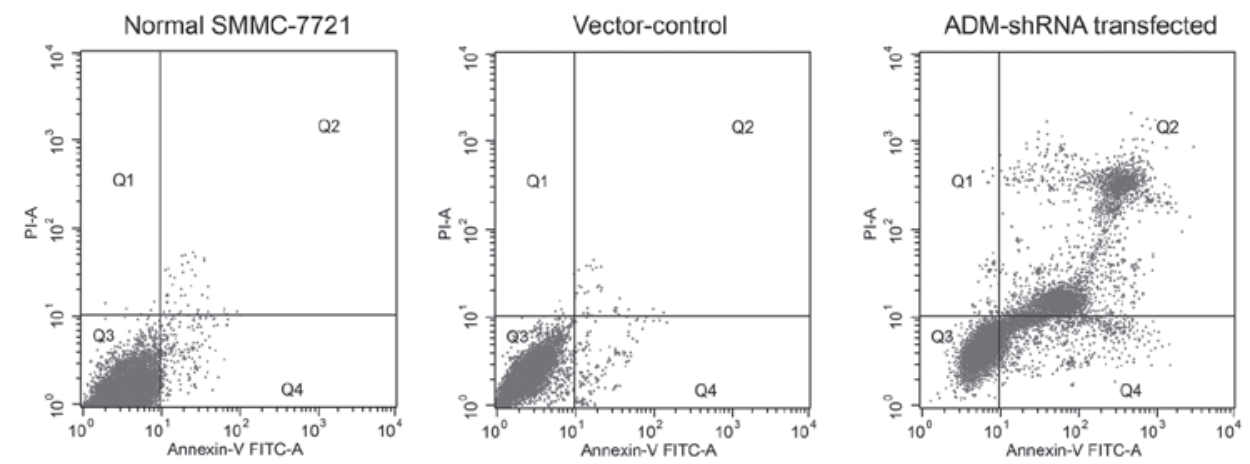

Figure 4. Gene silencing of ADM results in increased apoptosis compared with normal SMMC-7721 cells (left). SMMC-7721 cells were transfected with vector shRNA (middle) or ADM-shRNA (right). ADM, adrenomedullin; shRNA, short hairpin RNA; FITC, fluorescein isothiocyanate; PI, propidium iodide.

A

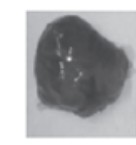

saline + PBS cisplatin + PBS

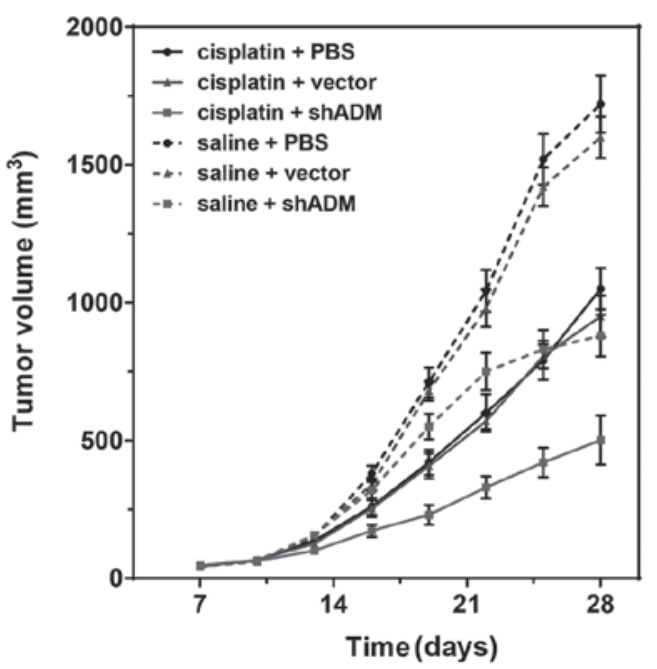

B

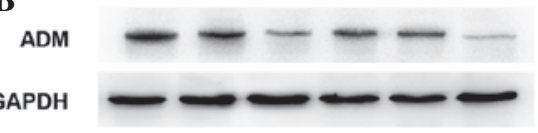

ADM

GAPDH

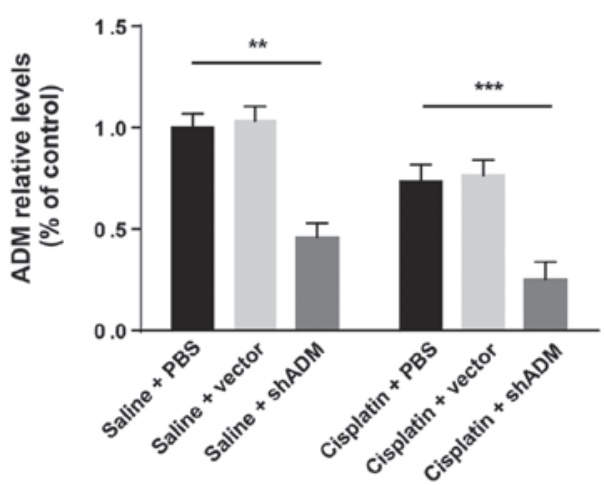

Figure 5. Therapeutic efficacy of cisplatin in combination with ADM-shRNA on reducing tumor growth in vivo. (A) Four representative subcutaneous tumors from different intratumoral injection therapies. Each group contained eight animals. The degree of tumor volume inhibition following each treatment is shown in the lower panel. (B) Mean ADM expression levels from randomly selected tumors from each treatment group exhibited a substantial decrease in the cisplatin + ADM-shRNA and saline + ADM-shRNA treatment groups. ${ }^{* *} \mathrm{P}<0.01$ and ${ }^{* * *} \mathrm{P}<0.0001$ compared with normal controls treated with PBS. ADM, adrenomedullin; shRNA, short hairpin RNA; PBS, phosphate-buffered saline.

effects on tumor ADM expression, with a significant interaction between these two factors $\left(\mathrm{F}_{2,30}=0.66 ; \mathrm{P}<0.05\right)$ (Fig. $\left.5 \mathrm{~B}\right)$. Consistent with the results regarding tumor growth, injection of ADM-shRNA plasmid significantly suppressed ADM expression in tumor tissue (saline plus shADM versus saline plus PBS, Bonferroni post-hoc, $\mathrm{P}<0.01$ ). Cisplatin combined with ADM-shRNA significantly inhibited tumor ADM expression, as compared with treatment with cisplatin alone (cisplatin plus shADM versus cisplatin plus PBS, Bonferroni post-hoc, $\mathrm{P}<0.0001)$. These findings support the hypothesis that the combination of cisplatin and ADM-shRNA can significantly suppress liver cancer tumor growth in vivo.

\section{Discussion}

The present study demonstrated that the expression of ADM in human HCC tissue was associated with the degree of malignancy and clinical prognosis. ADM levels were elevated under hypoxic conditions. SMMC-7721 cells showed an increased rate of cell death following inhibition of the expression of ADM using RNA interference. Furthermore, the inhibitory effect of cisplatin on tumor growth in vivo was significantly enhanced by combined treatment with ADM-shRNA. These results suggest that ADM may be a novel diagnostic and therapeutic target in HCC.

It was found in the present study that ADM mRNA expression in human HCC tissue is higher than that in adjacent normal liver tissue. This may result from hypoxia in situ, which has been shown to increase ADM mRNA expression in a variety of tumor cell types, including lung, breast, ovarian, colon and prostate cancer (16). Furthermore, it has been observed that hypoxia increases expression of ADM mRNA and secreted protein levels in a variety of tissues (17). These increases in ADM are at least partially mediated by the activation of HIF-1 
and VEGF $(18,19)$. The increased expression of ADM mRNA may result in increased angiogenic activity in tumor tissue (20). There is increasing evidence suggesting an important role for angiogenesis in HCC invasion and metastasis (21).

Hypoxia is a key microenvironmental regulatory factor in tumor growth that influences the progression of HCC by enhancing proliferation, angiogenesis and metastasis as well as chemo- and radio-resistance $(15,22)$. Evidence suggests that hypoxia affects the development and evolution of the tumor microenvironment by regulating the differentiation of tumor and stromal cells; thus, hypoxia plays a direct role in the maintenance of cancer stem cells and inhibition of differentiation of mesenchymal stem cells (23). Cancer cells adapt to hypoxic environments via the transcriptional activation of HIF-1 and VEGF, which activate the expression of genes involved in the glycolytic system, angiogenesis and cell survival (24). Consistent with previous results from Park et al (12), data in the present study showed that hypoxic conditions induced HIF-1, VEGF and ADM expression in three types of human HCC cell lines and that SMMC-7721 cells were the most sensitive to hypoxia. In human cancer cells, hypoxia-induced ADM signaling is enhanced predominantly via HIF-1, which is an important transcription factor produced in response to hypoxia. This transcription factor stimulates the synthesis of ADM and VEGF via hypoxic response elements located on their respective genes $(9,25)$. The increased ADM signaling has been demonstrated to enhance vascular smooth muscle cell maturation and thereby to promote tumor vessel growth (26). The growth of the tumor blood supply is essential in enabling tumor progression. The production and secretion of substances that affect vasodilation and angiogenesis may be necessary for tumor survival. Angiogenesis is closely associated with tumor prognosis, and, in clinical practice, cases with a high density of blood vessels have poor prognoses. Furthermore, ADM levels have been found to be correlated with the expression of $\mathrm{N}$-cadherin, which is an adhesion molecule that is involved in cell migration, invasion and metastasis of cancer cells (27). High levels of N-cadherin expression predict low recurrence-free and overall survival rates in patients with HCC (28), suggesting that the abnormal activation of ADM signaling in HCC tissue may also predict an unfavorable prognosis.

Evidence suggests that ADM exerts a wide range of effects on cell growth and apoptosis. These effects have been shown to be dependent on the cell type and experimental conditions (29). In tumor cells, ADM was found to inhibit apoptosis by upregulating B-cell lymphoma 2 and to increase cell growth and survival by activating oncogenic proteins, including Ras, Raf and protein kinase C (9). In HCC cells, signaling pathways independent of cyclic adenosine monophosphate and mitogen-activated protein kinases, including phosphinositide 3 kinase/Akt signaling, may have key roles in the regulation of cell proliferation and apoptosis induced by ADM (12). Given that ADM signaling was shown to accelerate HCC cell growth, an shRNA against ADM was used in the present study to investigate whether the inhibition of ADM expression in HCC cells suppresses hypoxia-induced tumor growth. Knockdown of ADM significantly inhibited cell proliferation and increased apoptosis under hypoxic stress conditions in SMMC-7721 cells. A previous study demonstrated that exogenous ADM antagonists or RNA interference specific for ADM expression effectively reduced ovarian cancer cell migration and osteosarcoma cell proliferation (30). Owing to the strong effect of ADM on angiogenesis and tumor growth in multiple tumor types, shRNA interference targeting ADM may be a powerful tool against oncogene expression and may be used therapeutically against several types of human cancer. In the present study, SMMC-7721 cells were transplanted into mice to generate subcutaneous tumors, and it was found that ADM-shRNA and cisplatin can act synergistically in vivo. These results further suggest the effectiveness of ADM shRNA in reducing HCC tumor growth.

In conclusion, the present study demonstrated that ADM is overexpressed in human HCC tissue compared with the tissue adjacent to the tumors. HCC cell proliferation was inhibited and HCC cell apoptosis was promoted by knocking down ADM using RNA interference. Treating tumor cells with ADM-shRNA combined with cisplatin inhibited HCC tumor growth to a greater extent than treatment with cisplatin alone. Therefore, the selective interruption of ADM signaling by shRNA may be an effective interventional therapeutic strategy in HCC.

\section{References}

1. Moradpour D and Blum HE: Pathogenesis of hepatocellular carcinoma. Eur J Gastroenterol Hepatol 17: 477-483, 2005.

2. Tanaka S and Arii S: Current status and perspective of antiangiogenic therapy for cancer: hepatocellular carcinoma. Int J Clin Oncol 11: 82-89, 2006.

3. Gwak GY, Yoon JH, Kim KM, Lee HS, Chung JW and Gores GJ: Hypoxia stimulates proliferation of human hepatoma cells through the induction of hexokinase II expression. J Hepatol 42: 358-364, 2005.

4. Liotta LA, Steeg PS and Stetler-Stevenson WG: Cancer metastasis and angiogenesis: an imbalance of positive and negative regulation. Cell 64: 327-336, 1991.

5. Hanahan D and Folkman J: Patterns and emerging mechanisms of the angiogenic switch during tumorigenesis. Cell 86: 353-364, 1996.

6. Carmeliet P and Jain RK: Angiogenesis in cancer and other diseases. Nature 407: 249-257, 2000.

7. Dvorak HF: Vascular permeability factor/vascular endothelial growth factor: a critical cytokine in tumor angiogenesis and a potential target for diagnosis and therapy. J Clin Oncol 20: 4368-4380, 2002.

8. Kitamura K, Kangawa K, Kawamoto M, et al: Adrenomedullin: a novel hypotensive peptide isolated from human pheochromocytoma. Biochem Biophys Res Commun 192: 553-560, 1993.

9. Nikitenko LL, Fox SB, Kehoe S, Rees MC and Bicknell R: Adrenomedullin and tumour angiogenesis. Br J Cancer 94: 1-7, 2006.

10. Jiménez N, Abasolo I, Jongsma J, et al: Androgen-independent expression of adrenomedullin and peptidylglycine alpha-amidating monooxygenase in human prostatic carcinoma. Mol Carcinog 38: 14-24, 2003.

11. Li Z, Takeuchi S, Otani T and Maruo T: Implications of adrenomedullin expression in the invasion of squamous cell carcinoma of the uterine cervix. Int J Clin Oncol 6: 263-270, 2001.

12. Park SC, Yoon JH, Lee JH, et al: Hypoxia-inducible adrenomedullin accelerates hepatocellular carcinoma cell growth. Cancer Lett 271: 314-322, 2008.

13. Edmondson HA and Steiner PE: Primary carcinoma of the liver: a study of 100 cases among 48,900 necropsies. Cancer 7: 462-503, 1954.

14. Deville JL, Salas S, Figarella-Branger D, et al: Adrenomedullin as a therapeutic target in angiogenesis. Expert Opin Ther Targets 14: 1059-1072, 2010.

15. Wu SD, Ma YS, Fang Y, Liu LL, Fu D and Shen XZ: Role of the microenvironment in hepatocellular carcinoma development and progression. Cancer Treat Rev 38: 218-225, 2012.

16. Zudaire E, Martínez A and Cuttitta F: Adrenomedullin and cancer. Regul Pept 112: 175-183, 2003. 
17. Oehler MK, Norbury C, Hague S, Rees MC and Bicknell R: Adrenomedullin inhibits hypoxic cell death by upregulation of Bcl-2 in endometrial cancer cells: a possible promotion mechanism for tumour growth. Oncogene 20: 2937-2945, 2001.

18. Oladipupo S, Hu S, Kovalski J, et al: VEGF is essential for hypoxia-inducible factor-mediated neovascularization but dispensable for endothelial sprouting. Proc Natl Acad Sci USA 108: 13264-13269, 2011.

19. Frede S, Freitag P, Otto T, Heilmaier C and Fandrey J: The proinflammatory cytokine interleukin lbeta and hypoxia cooperatively induce the expression of adrenomedullin in ovarian carcinoma cells through hypoxia inducible factor 1 activation. Cancer Res 65: 4690-4697, 2005.

20. Ribatti D, Nico B, Spinazzi R, Vacca A and Nussdorfer GG: The role of adrenomedullin in angiogenesis. Peptides 26: 1670-1675, 2005.

21. Jung KH, Zheng HM, Jeong Y, et al: Suppression of tumor proliferation and angiogenesis of hepatocellular carcinoma by HS-104, a novel phosphoinositide 3-kinase inhibitor. Cancer Lett 328: $176-187,2013$.

22. Harris AL: Hypoxia - a key regulatory factor in tumour growth. Nat Rev Cancer 2: 38-47, 2002.

23. Kim Y, Lin Q, Glazer PM and Yun Z: Hypoxic tumor microenvironment and cancer cell differentiation. Curr Mol Med 9: 425-434, 2009.
24. Tang N, Wang L, Esko J, et al: Loss of HIF-1alpha in endothelial cells disrupts a hypoxia-driven VEGF autocrine loop necessary for tumorigenesis. Cancer Cell 6: 485-495, 2004

25. Carmeliet P, Dor Y, Herbert JM, et al: Role of HIF-1alpha in hypoxia-mediated apoptosis, cell proliferation and tumour angiogenesis. Nature 394: 485-490, 1998.

26. Iwase T, Nagaya N, Fujii T, et al: Adrenomedullin enhances angiogenic potency of bone marrow transplantation in a rat model of hindlimb ischemia. Circulation 111: 356-362, 2005.

27. Hazan RB, Phillips GR, Qiao RF, Norton L and Aaronson SA: Exogenous expression of $\mathrm{N}$-cadherin in breast cancer cells induces cell migration, invasion, and metastasis. J Cell Biol 148: 779-790, 2000.

28. Gwak GY, Yoon JH, Yu SJ, et al: Anti-apoptotic N-cadherin signaling and its prognostic implication in human hepatocellular carcinomas. Oncol Rep 15: 1117-1123, 2006.

29. Shichiri M and Hirata Y: Regulation of cell growth and apoptosis by adrenomedullin. Hypertens Res 26 (Suppl): S9-S14, 2003.

30. Pang X, Shang H, Deng B, Wen F and Zhang Y: The interaction of adrenomedullin and macrophages induces ovarian cancer cell migration via activation of RhoA signaling pathway. Int J Mol Sci 14: 2774-2787, 2013. 